\title{
A Wilkinson Power Divider with Harmonic Suppression and Size Reduction using High-low Impedance Resonator Cells
}

\author{
Mohsen HAYATI, Ashkan ABDIPOUR, Arash ABDIPOUR
}

Electrical Engineering Dept., Faculty of Engineering, Razi University, Tagh-E-Bostan, Kermanshah-67149, Iran

mohsen_hayati@yahoo.com, Ashkan_abdipour@yahoo.com, Arash.abdipour@yahoo.com

\begin{abstract}
A miniaturized Wilkinson power divider using high-low impedance resonator cells is designed and fabricated. The proposed power divider occupies $23.7 \%$ of the conventional structure circuit area at the operating frequency of $0.9 \mathrm{GHz}$ and it is also able to suppress harmonics. According to the measured results at $0.9 \mathrm{GHz}$, the insertion-losses of output ports are $3.087 \mathrm{~dB}$, the returnlosses at all ports are more than $30 \mathrm{~dB}$, and the isolation between output ports is better than $35 \mathrm{~dB}$. Also, $2^{\text {nd }}$ to $10^{\text {th }}$ spurious frequencies are suppressed. According to the measured $S_{11}$, when it is less than $-15 \mathrm{~dB}$ (from $0.65 \mathrm{GHz}$ to $1.1 \mathrm{GHz}$ ) the fractional bandwidth of the proposed structure is $50 \%$. Good agreement between simulation and measured results is achieved.
\end{abstract}

\section{Keywords}

Harmonic suppression, high-low impedance resonator, miniaturized Wilkinson power divider

\section{Introduction}

The power dividers are extremely important devices in microwave and millimeter-wave systems such as mixers, frequency multipliers, and the feeding network for an antenna array. There are several approaches for size reduction and harmonic suppression in the process of designing power dividers. The reported Wilkinson power divider with conventional quarter-wavelength transmission-line (TLIN) in [1], occupies a large area (especially at low operating frequencies) and it is not capable of suppressing spurious frequencies. In order to overcome these disadvantages several methods are used to reduce overall circuit size and suppress unwanted harmonics in the Wilkinson power dividers [2-7]. For instance, for size reduction and harmonic suppression, a kind of Wilkinson power divider based on standard printed-circuit-board (PCB) etching processes is reported in [2]. This structure is designed considering slow-wave loading and reduced the occupied area to $36.5 \%$ of the conventional structure at its operating frequency. In [3] and [4], microstrip electromagnetic band- gap (EBG) structure has been applied to the conventional power divider design, which led to the miniaturization and harmonic rejection in the conventional Wilkinson power divider.

Utilizing defected ground structure (DGS) can reject unwanted harmonics and decrease the occupied area in power dividers as it has been reported in [5] and [6]. Since, (DGS) and (EBG) need etching process, so their fabrication process is complex and these methods are not useable on a metal surface. The use of $\pi$-equivalent shunt-stubbased artificial transmission lines can effectively decrease the circuit size of conventional power divider [7]. This structure occupies $14.7 \%$ of the conventional power divider, but it is not capable for harmonic rejection at its operating frequency of $0.9 \mathrm{GHz}$.

In this paper, a Wilkinson power divider with harmonic suppression and size reduction is proposed. In order to reduce the circuit size of the conventional Wilkinson power divider, transmission lines with high-low impedance resonators are used instead of conventional quarter-wavelength TLIN sections. This technique not only reduces the occupied area to $23.7 \%$ of conventional one at operating frequency of $0.9 \mathrm{GHz}$, but also suppresses the second up to tenth harmonics.

\section{Power Divider Design}

\subsection{The Procedure of Designing and the Effect of High-low Impedance Resonator on Size Reduction and Harmonic Sup- pression}

Utilizing traditional TLIN in the structure of power dividers results in a large occupied area. Furthermore, this kind of transmission line is not able to suppress spurious frequencies. Using transmission line with loaded capacitance instead of quarter-wavelength transmission-line not only reduces the circuit size, but also can suppress unwanted harmonics. In the first step, a conventional Wilkinson power divider with an operating frequency at $0.9 \mathrm{GHz}$ 
is designed as it is shown in Fig. 1a. In order to make capacitor loading on each $\lambda / 4$ TLIN, four resonators with primary dimensions of $\mathrm{W} 1=\mathrm{W} 2=0.1 \mathrm{~mm}, \mathrm{~L} 1=\mathrm{L} 2=$ $0.1 \mathrm{~mm}, \mathrm{~W} 3=0.1 \mathrm{~mm}$ and $\mathrm{L} 3=0.1 \mathrm{~mm}$ are added inside the free area of the conventional structure. These values are selected to control the effects of changing dimensions on frequency response and determine the operating frequency. The locations of the added resonators are determined with a, b, c and d in Fig. 1b. By increasing the values of W1, W2, L1 and L2 as low impedance TLINs a large loaded capacitance can be obtained. In order to reduce the occupied area of the power divider, the length of the main TLIN can decrease simultaneously, with increasing the dimensions of low impedance TLINs. Note that changing the values of variables does not have to shift the desired operating frequency, i.e. $0.9 \mathrm{GHz}$. Furthermore, adding these resonators makes a lowpass filter on the each main transmission line of the designed circuit. It appears in the insertion loss (S21), because of high order harmonics suppression in the frequency response.

The proposed power divider at $0.9 \mathrm{GHz}$ and its equivalent circuit using lumped components are shown in Fig. 2a and Fig. 2b, respectively. In Fig. $2 \mathrm{~b} L_{\mathrm{b}}, L_{\mathrm{c}}$ and $L_{\mathrm{d}}$ are equivalent inductors caused by the main transmission line. High-low impedance resonators are modeled by $L_{\mathrm{a}}$, $C 1$ and $C 2$, where $L_{\mathrm{a}}$ determines high impedance transmission lines of these resonators. $C 1$ and $C 2$ show low impedance open-circuit transmission lines of resonators 1 and 2, respectively. The gaps $g_{1}, g_{2}$ and $g_{3}$ between low impedance open-circuit lines cause coupling effects, which are modeled by $C_{\mathrm{g} 1}, C_{\mathrm{g} 2}$ and $C_{\mathrm{g} 3}$. Furthermore, $C_{\mathrm{p} 1}$ and $C_{\mathrm{p} 2}$ present the capacitance between the microstrip structure and the ground. Lout accounts for inductor of output transmission lines. Notice that the coupling capacitances between the main transmission line and open-circuit transmission lines are not included in the LC circuit as they are trivial.

Open-stub loads in the structure of high-low impedance resonators of the proposed power divider, modeled by $C 1$ and $C 2$ lead to a large shunt capacitance. Therefore, the circuit size of the proposed Wilkinson power divider could reduce because the propagation constant, i.e. $\beta$ enhances ( $\beta$ proposed $/ \beta$ conventional is about 1.923 ). The relationship for $\beta$ is given by:

$$
\begin{gathered}
\beta=\omega \sqrt{L C}, \\
\beta=\frac{2 \pi}{\lambda_{\mathrm{g}}}
\end{gathered}
$$

where in (1a) $L$ is the total inductance in per length unit of the main transmission line and high impedance line, and $C$ depicts the total capacitance in per length unit of the main transmission line. In (1b) $\lambda_{\mathrm{g}}$ determines guided wavelength. Since $C$ (the capacitance of proposed power divider) is increased in comparison with the transmission line of a conventional power divider, the propagation constant is enhanced considerably. As a result, the occupied area of circuit will be decreased [1].

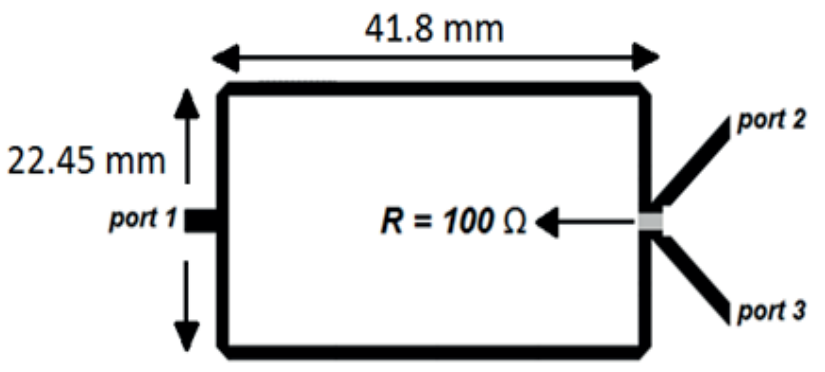

Fig. 1a. Topology of conventional Wilkinson power divider at operating frequency equal to $0.9 \mathrm{GHz}$.

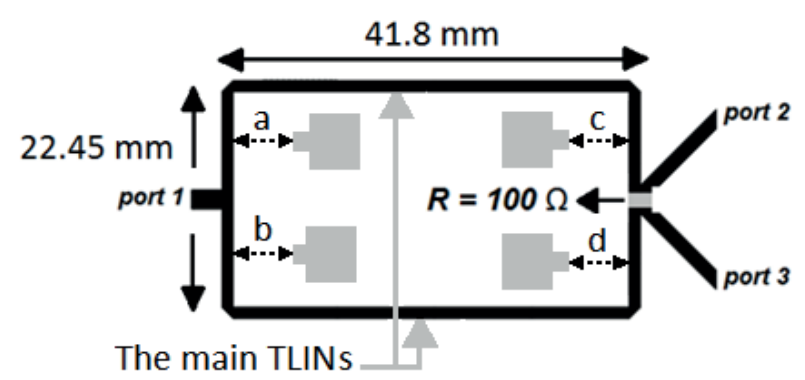

Fig. 1b. Topology of conventional Wilkinson power divider at operating frequency of $0.9 \mathrm{GHz}$ with the locations of added high-low impedance resonators.

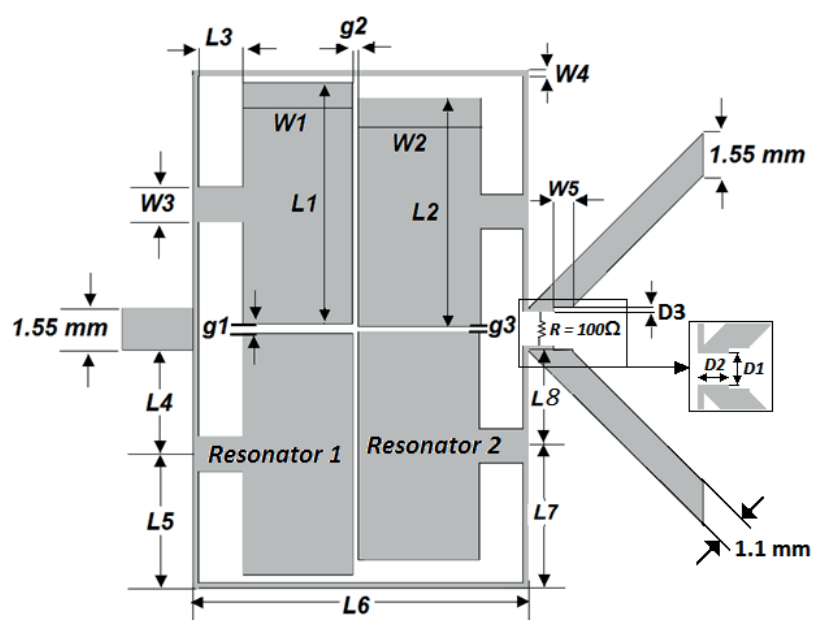

Fig. 2a. Topology of the proposed Wilkinson power divider.

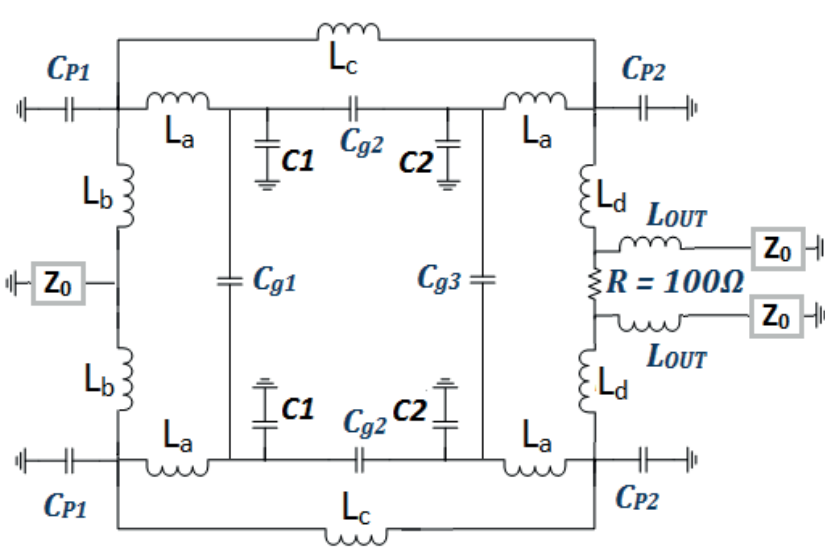

Fig. 2b. Equivalent LC circuit of the proposed Wilkinson power divider. 
Moreover, based on insertion loss (S21) of the proposed structure shown in Fig. 6, optimized transmission line with high-low impedance resonators in higher frequencies has features of a lowpass filter. Spurious resonant frequencies of the resonator have been shifted by the highlow impedance resonators from the integer multiples of the basic resonant frequency [1]. So, replacing conventional quarter-wavelength transmission-line with a transmission line loaded by the high-low impedance resonators leads to harmonic suppression.

\subsection{The Structure of the Proposed Power Divider}

Comparison between the topology of conventional power divider in Fig. 1a and the proposed design, illustrated in Fig. 2a shows that in the proposed power divider, four microstrip high-low impedance resonator cells are used within the free area of the conventional Wilkinson power divider. The low impedance patches with rectangular shapes are microstrip open-stubs, so each of the highlow impedance resonator cells refers to a loaded capacitor. As a result, the capacitor loading not only reduces the circuit size, but also can suppress spurious frequencies.

The designed resonators, i.e. resonators 1 and 2 in both sides (left and right) have the same structure, but their rectangular patches have different dimensions. The circuit size of the proposed power divider and the conventional structure are $18.55 \mathrm{~mm} \times 12 \mathrm{~mm}$ and $41.8 \mathrm{~mm} \times$ $22.45 \mathrm{~mm}$, respectively. It shows that the occupied area is reduced to $23.7 \%$ of the conventional power divider at operating frequency of $0.9 \mathrm{GHz}$. The type of the used $100 \Omega$ isolation resistor is 0603 , which is placed between two output ports. The dimensions of the proposed power divider shown in Fig. $2 \mathrm{a}$ are: $\mathrm{W} 1=3.9, \mathrm{~W} 2=4.3, \mathrm{~W} 3=$ $1.2, \mathrm{~W} 4=0.2, \mathrm{~W} 5=0.66, \mathrm{D} 1=1.15, \mathrm{D} 2=1.1, \mathrm{D} 3=0.2$, $\mathrm{L} 1=8.6, \mathrm{~L} 2=8.2, \mathrm{~L} 3=1.6, \mathrm{~L} 4=3.7, \mathrm{~L} 5=4.8, \mathrm{~L} 6=12$, $\mathrm{L} 7=5.1, \mathrm{~L} 8=3.4, \mathrm{~g} 1=0.3, \mathrm{~g} 2=0.2$ and $\mathrm{g} 3=0.15$ (all in millimeter). The calculated values of inductors and capacitors of the shown LC circuit in Fig. $2 \mathrm{~b}$ are [8]: $\mathrm{La}=$ $0.438 \mathrm{nH}, \mathrm{Lb}=1.838 \mathrm{nH}, \mathrm{Lc}=12.38 \mathrm{nH}, \mathrm{Ld}=1.68 \mathrm{nH}$, $\mathrm{C} 1=1.36 \mathrm{pF}, \mathrm{C} 2=1.43 \mathrm{pF}, \mathrm{Cg} 1=25 \mathrm{fF}, \mathrm{Cg} 2=128 \mathrm{fF}$, $\mathrm{Cg} 3=42 \mathrm{fF}, \mathrm{Cp} 1=0.471 \mathrm{pF}, \mathrm{Cp} 2=0.466 \mathrm{pF}$. Note that the values of $\mathrm{Cg} 1, \mathrm{Cg} 2$ and $\mathrm{Cg} 3$ are achieved by tuning. Comparison between LC simulation and EM simulation results of the shown circuits in Figs. $2 a$ and $b$ on a substrate with permittivity of 2.2 , thickness of $0.508 \mathrm{~mm}$ and loss tangent of 0.0009 are depicted in Figs. 3-6.

\section{Simulated and Measured Results}

The measured and simulated results of the proposed power divider are accomplished using Agilent's ADS Electromagnetic simulator (EM Simulator) software and HP 8720B vector network analyzer, respectively. The operating frequency of the proposed structure is located at $0.9 \mathrm{GHz}$. The designed microstrip Wilkinson power

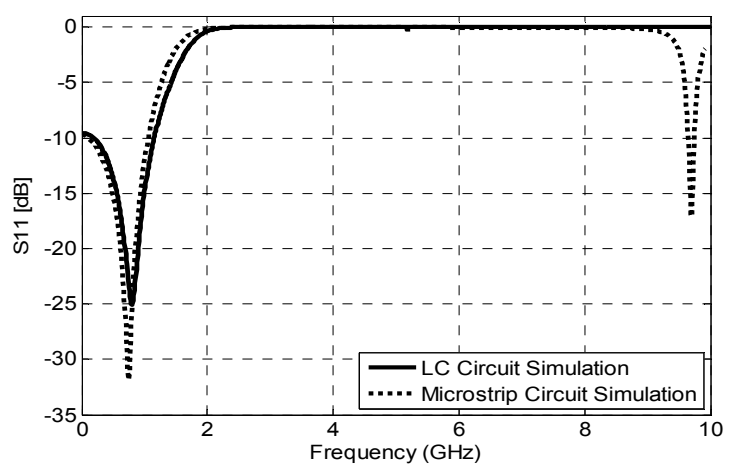

Fig. 3. Comparison between LC simulation and EM simulation results of input return-loss.

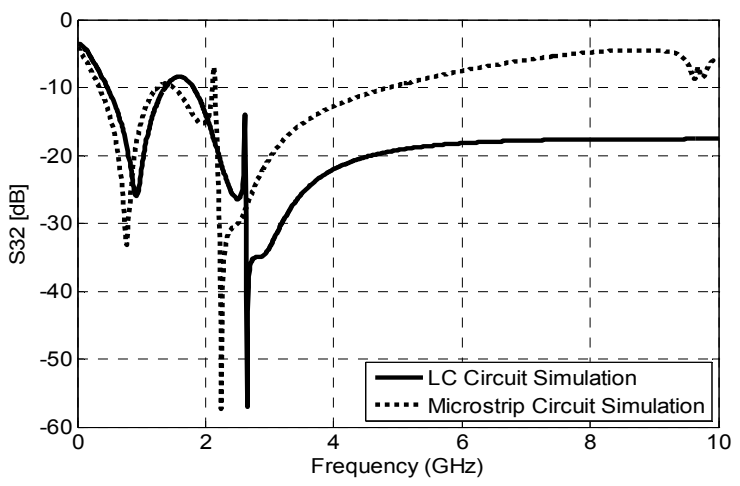

Fig. 4. Comparison between LC simulation and EM simulation results of isolation.

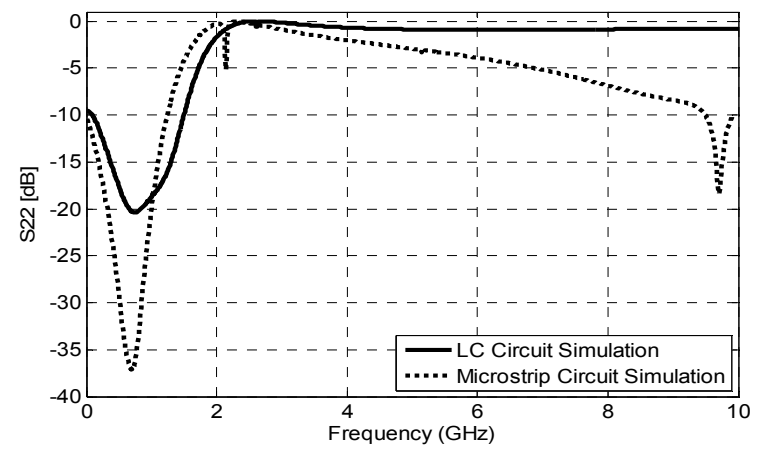

Fig. 5. Comparison between LC simulation and EM simulation results of output return-loss.

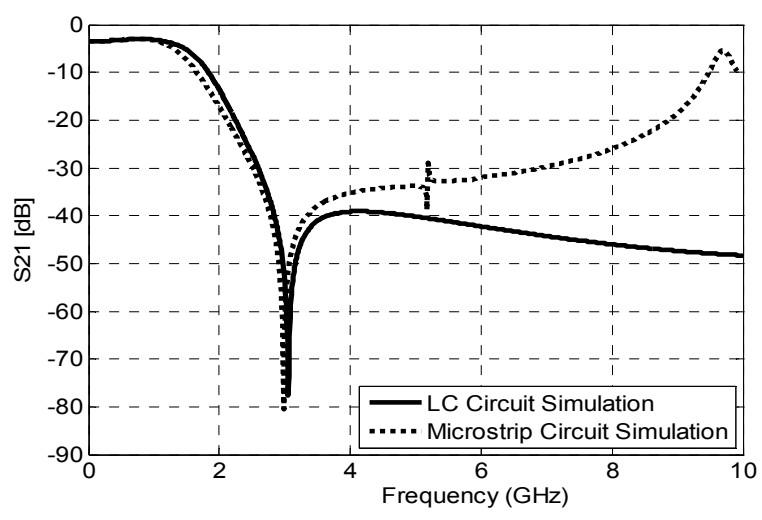

Fig. 6. Comparison between LC simulation and EM simulation results of insertion-loss. 
divider is fabricated on RT/Duroid 5880 substrate with the thickness of $0.508 \mathrm{~mm}$, the permittivity of 2.2 and the loss tangent of 0.0009 . The results of measurement and simulation of S-parameters are illustrated in Figs. 7-11. As it is shown in Fig. 7, the measured return loss (S11) is at least $-15 \mathrm{~dB}$ from $0.65 \mathrm{GHz}$ to $1.1 \mathrm{GHz}$. In Fig. 8, the measurement shows over the frequency range $0.66-1.12 \mathrm{GHz}$ the isolation (S23) is better than $-15 \mathrm{~dB}$. According to Fig. 9, the output return loss (S22) less than $-15 \mathrm{~dB}$ from $0.28 \mathrm{GHz}$ to $1.25 \mathrm{GHz}$ is achieved. It can be observed from the measured insertion loss (S21) in Fig. 10, both even and odd spurious harmonics from $1.8 \mathrm{GHz}$ to $9 \mathrm{GHz}$, i.e. second to tenth harmonics have been suppressed, where the $3^{\text {rd }}$ to $10^{\text {th }}$ harmonics are suppressed with a level less than $-20 \mathrm{~dB}$ and the second harmonic is suppressed with a level better than $-11 \mathrm{~dB}$. It is to be noted that the suppression of higher order harmonic frequencies is related to S21 and S31. Exactly at operating frequency equal to $0.9 \mathrm{GHz}$, the measured $\mathrm{S} 11, \mathrm{~S} 32$, and $\mathrm{S} 22$ are $-33 \mathrm{~dB},-38.88 \mathrm{~dB}$ and $-48 \mathrm{~dB}$, respectively. Furthermore, the measured insertion loss shows that $\mathrm{S} 21$ at $0.9 \mathrm{GHz}$ is $-3.087 \mathrm{~dB}$. The characteristic impedance of all three ports are $50 \Omega$. Tab. 1 shows the comparison between the proposed power divider and the other published works. Based on the results of measurement shown in Fig. 11, an appropriate phase performance between two output ports around operating frequency is achieved. The measured phase difference of ports 2 and 3 as output ports is $\pm 0.15^{\circ}$. It shows that the proposed Wilkinson power divider is symmetric, so $|\mathrm{S} 21|=|\mathrm{S} 31|$ (thus, harmonic suppression is related to both S21 and S31) and $|\mathrm{S} 22|=|\mathrm{S} 33|$. The photograph of the proposed Wilkinson power divider is shown in Fig. 12.

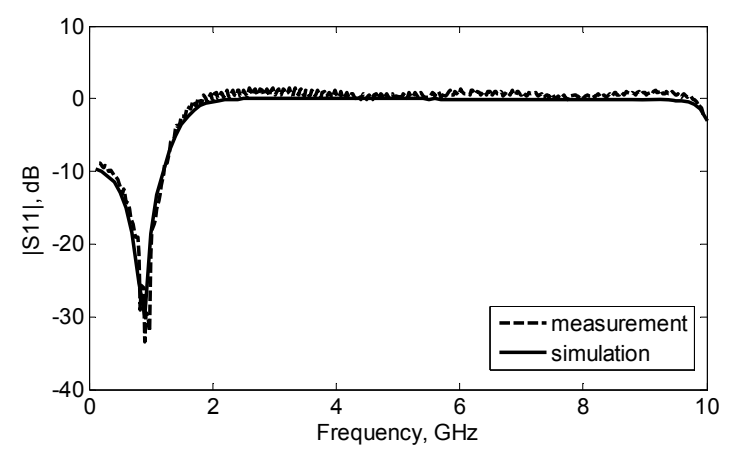

Fig. 7. Simulated and measured input return-loss.

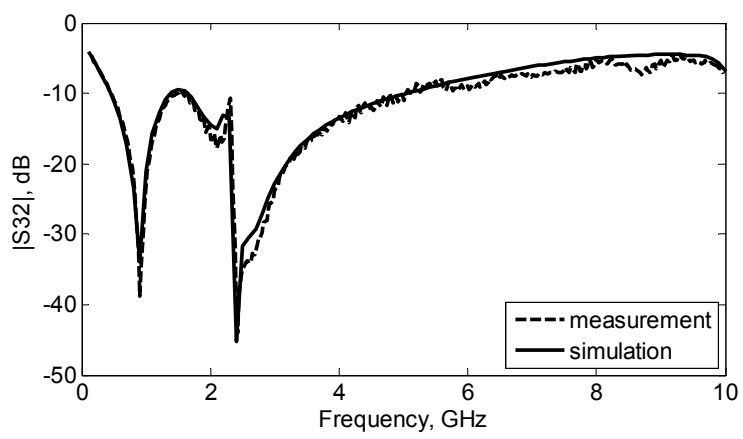

Fig. 8. Simulated and measured isolation.

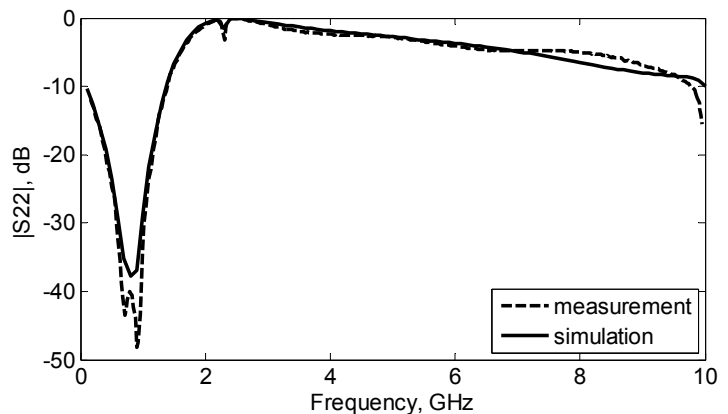

Fig. 9. Simulated and measured output return-loss.

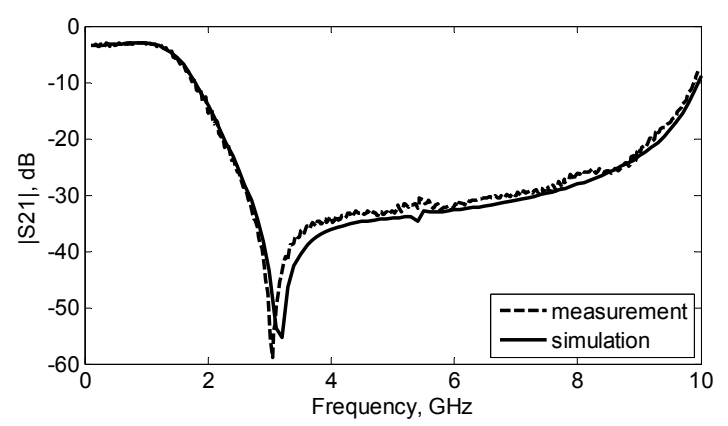

Fig. 10. Simulated and measured insertion-loss.

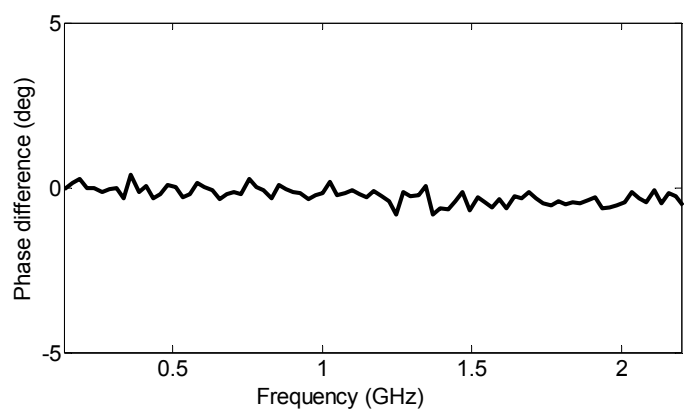

Fig. 11. Measured phase difference between S21 and S31 of the proposed power divider.

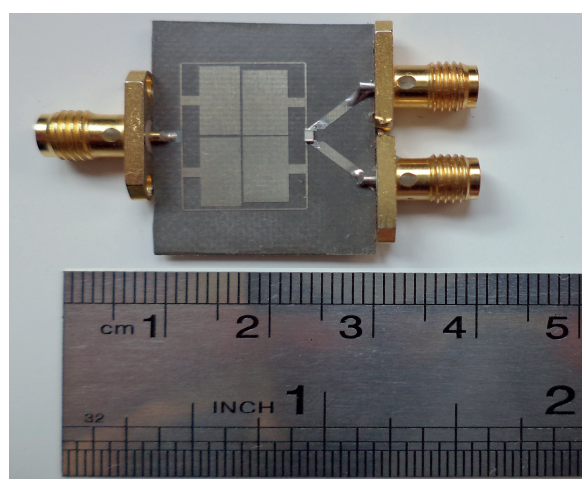

Fig. 12. Photograph of the proposed structure.

\section{Conclusion}

In this paper, a Wilkinson power divider using highlow impedance resonator cells for harmonic suppression and size reduction is proposed. The key features of the proposed structure are: 


\begin{tabular}{|c|c|c|c|c|c|c|c|c|c|c|}
\hline \multirow{2}{*}{ Ref. } & \multirow{2}{*}{ Area reduction } & \multicolumn{9}{|c|}{ Harmonic suppression (dB) } \\
\cline { 3 - 11 } & & $2^{\text {nd }}$ & $3^{\text {rd }}$ & $4^{\text {th }}$ & $5^{\text {th }}$ & $6^{\text {th }}$ & $7^{\text {th }}$ & $8^{\text {th }}$ & $9^{\text {th }}$ & $10^{\text {th }}$ \\
\hline$[2]$ & $63 \%$ & 13 & 29 & 32 & 34 & - & - & - & - & - \\
\hline$[3]$ & $70 \%$ & 8 & 32 & 10 & 12 & - & - & - & - & - \\
\hline$[4]$ & $39 \%$ & 26 & 25 & - & - & - & & - & - & - \\
\hline$[5]$ & $10 \%$ & 18 & 15 & - & - & - & & - & - & - \\
\hline$[6]$ & $66 \%$ & 13 & 35 & - & - & - & & - & - & - \\
\hline$[7]$ & $85.3 \%$ & - & - & - & - & - & & - & - & - \\
\hline This work & $76.3 \%$ & 11.3 & 31.5 & 35.5 & 33.2 & 32.4 & 30.1 & 25.9 & 25.9 & 22.4 \\
\hline
\end{tabular}

Tab. 1. Comparison between the performance of the proposed power divider and previous works.

1- Small occupied area, i.e. $18.55 \mathrm{~mm} \times 12 \mathrm{~mm}$ at the frequency of $0.9 \mathrm{GHz}$;

2- At operating frequency low insertion-losses of output ports $(3.087 \mathrm{~dB})$ and more than $30 \mathrm{~dB}$ return-losses at all ports are obtained. Moreover, better than $35 \mathrm{~dB}$ isolation and $\pm 0.15^{\circ}$ phase difference between output ports are achieved;

3- In the proposed structure spurious frequencies from $1.8 \mathrm{GHz}$ up to $9 \mathrm{GHz}$, i.e. second to tenth harmonics are suppressed.

Therefore, the designed circuit with its operating frequency at $0.9 \mathrm{GHz}$ can be used, where a power divider with small size and capable of suppress harmonics is required.

\section{References}

[1] POZAR, D. M. Microwave Engineering. 3rd ed. New York: Wiley, 2005, ch. 7, p. 333-337.

[2] WANG, J., NI, J., GUO, Y. X., FANG, D. Miniaturized microstrip Wilkinson power divider with harmonic suppression. IEEE Microwave and Wireless Components Letters, 2009, vol. 19, no. 7, p. 440-442. DOI: 10.1109/LMWC.2009.2022124

[3] LIN, C. M., SU, H. H., CHIU, J. C., WANG, Y. H. Wilkinson power divider using microstrip EBG cells for the suppression of harmonics. IEEE Microwave and Wireless Components Letters, 2007, vol. 17, no. 10, p. 700-702. DOI: 10.1109/LMWC.2007.905595

[4] ZHANG, F., LI, C. F. Power divider with microstrip electromagnetic band gap element for miniaturization and harmonic rejection. Electronics Letters, 2008, vol. 44, no. 6, p. 422-423. DOI: 10.1049/el:20083693

[5] WOO, D. J., LEE, T. K. Suppression of harmonics in Wilkinson power divider using dual-band rejection by asymmetric DGS. IEEE Transactions on Microwave Theory and Techniques, 2005, vol. 53, no. 6, p. 2139-2144. DOI: 10.1109/TMTT.2005.848772

[6] YANG, J., GU, C. F., WU, W. Design of novel compact coupled microstrip power divider with harmonic suppression. IEEE Microwave and Wireless Components Letters, 2008, vol. 18, no. 9, p. 572-574. DOI: 10.1109/LMWC.2008.2002444
[7] TSENG, C.-H., WU, C.-H. Compact planar Wilkinson power divider using pi-equivalent shunt-stub-based artificial transmission lines. Electronics Letters, 2010, vol. 46, no. 19, p. 1327-1328. DOI: $10.1049 / \mathrm{el} .2010 .2194$

[8] HONG, J.-S., LANCASTER, M. J. Microstrip Filters for RF/Microwave Applications. John Wiley \& Sons, Inc., 2001.

\section{About the Authors ...}

Mohsen HAYATI received the BE in Electronics and Communication Engineering from Nagarjuna University, India, in 1985, and the ME and PhD in Electronics Engineering from Delhi University, Delhi, India, in 1987 and 1992, respectively. He joined the Electrical Engineering Dept., Kermanshah Branch, Islamic Azad University, Kermanshah, as a part time assistant professor in 2004. At present, he is a professor with the Electrical Engineering Dept., Kermanshah Branch, Islamic Azad University. He has published more than 155 papers in international and domestic journals and conferences. His current research interests include microwave and millimeter wave devices and circuits, application of computational intelligence, artificial neural networks, fuzzy systems, neuro-fuzzy systems, electronic circuit synthesis, modeling and simulations.

Ashkan ABDIPOUR received the B.S in Electronics Engineering from Islamic Azad University, Kermanshah Branch, Kermanshah, Iran, in 2009 and M.S degree from the Razi University, Kermanshah, Iran, in 2013. His research interests include microwave and millimeter wave devices and circuits.

Arash ABDIPOUR received the B.S in Electronics Engineering from Islamic Azad University, Kermanshah Branch, Kermanshah, Iran, in 2009 and M.S degree in Electronic Engineering from the University of Science and Research, Kermanshah Branch, Kermanshah, Iran in 2013. His research interests include microwave and millimeter wave devices and circuits. 